\title{
Congress, conferences, workshops and courses
}

August 2016

28 August-02 September 2016

Firenze, Italy

36th International Symposium on Halogenated Persistent Organic Pollutants - Dioxin 2016

e-mail: info@dioxin2016firenze.org

http://dioxin2016firenze.org

September 2016

11-15 September 2016

Seville, Spain

6th EuCheMS Chemistry Congress

http://euchems-seville2016.eu

12-15 September 2016

Ghent, Belgium

18th International Conference on Heavy Metals in the Environment - ICHMET 2016

e-mail: ichmet2016@ugent.be

http://www.ichmet2016.ugent.be

18-21 September 2016

Dallas, TX, USA

AOAC Annual Meeting and Exhibition

e-mail: Lchelf@aoac.org

http://www.aoac.org
October 2016

12-13 October 2016

Geel, Belgium

JRC-IRMM Training Course: Use of Reference Materials and the Estimation of Measurement Uncertainty e-mail: jrc-irmm-rm-course @ec.europa.eu https://ec.europa.eu/jrc

January 2017

23 January 2017

Tel Aviv, Israel

International Workshop on Validation of Test Methods, Human Errors and Measurement Uncertainty of Results

e-mail: reutl@bioforum.co.il

http://www.isranalytica.org.il

24-25 January 2017

Tel Aviv, Israel

Isranalytica Conference and Exhibition

e-mail: reutl@bioforum.co.il

http://www.isranalytica.org.il 